\title{
Intensity scaling limitations of laser-driven proton acceleration in the TNSA-regime
}

\author{
S. Keppler,${ }^{1,2,3}$ N. Elkina, ${ }^{1,3}$ G. A. Becker, ${ }^{2}$ J. Hein,,${ }^{1,2}$ M. Hornung $\odot,{ }^{1,2,3}$ M. Mäusezahl $\odot,{ }^{1}$ \\ C. Rödel, ${ }^{1,2,3}$ I. Tamer, ${ }^{1,2,3}$ M. Zepf,${ }^{1,2,3}$ and M. C. Kaluza ${ }^{1,2,3,}$ \\ ${ }^{1}$ Helmholtz Institute Jena, Fröbelstieg 3, 07743 Jena, Germany \\ ${ }^{2}$ Institute of Optics and Quantum Electronics, Max-Wien Platz 1, 07743 Jena, Germany \\ ${ }^{3}$ GSI Helmholtzzentrum für Schwerionenforschung, D-64291 Darmstadt, Germany
}

(Received 21 July 2020; revised 26 March 2021; accepted 6 December 2021; published 31 January 2022)

\begin{abstract}
We report on experimental results on laser-driven proton acceleration using high-intensity laser pulses. We present power law scalings of the maximum proton energy with laser pulse energy and show that the scaling exponent $\xi$ strongly depends on the scale length of the preplasma, which is affected by the temporal intensity contrast. At lower laser intensities, a shortening of the scale length leads to a transition from a square root toward a linear scaling. Above a certain threshold, however, a significant deviation from this scaling is observed. Two-dimensional particle-in-cell simulations show that, in this case, the electric field accelerating the ions is generated earlier and has a higher amplitude. However, since the acceleration process starts earlier as well, the fastest protons outrun the region of highest field strength, ultimately rendering the acceleration less effective. Our investigations thus point to a principle limitation of the proton energy in the target normal sheath acceleration regime, which would explain why a significant increase of the maximum proton energy above the limit of $100 \mathrm{MeV}$ has not yet been achieved.
\end{abstract}

DOI: 10.1103/PhysRevResearch.4.013065

\section{INTRODUCTION}

The availability of proton pulses accelerated to kinetic energies well above $10 \mathrm{MeV}$ during laser-plasma interactions bears great potential for applications in fundamental science, inertial fusion energy $[1,2]$, time-resolved radiography of plasmas $[3,4]$, as a frontend for conventional accelerators [5-7], for material sciences [8-10], and radiation therapy $[11,12]$. While plasma-accelerated, multi-10-MeV protons are already used for applications [13], energies in excess of $200 \mathrm{MeV}$ are required, e.g., for radiation therapy. To further increase the proton energy, the driving-laser technology is continuously improved and acceleration schemes are investigated. These include radiation pressure acceleration [14], collisionless shock acceleration $[15,16]$, or mechanisms dominant in the near-critical density or induced-transparency regime $[17,18]$. To date, the highest energies $(\approx 100 \mathrm{MeV})$ were achieved via acceleration during the onset of relativistically induced transparency from nanometer-thin foils [19].

In most experiments, however, the target normal sheath acceleration (TNSA) mechanism [20] is dominant. Here, relativistic electrons are generated during the interaction of a high-intensity laser with preplasma formed at the front side of a solid target - typically a thin foil. Such preplasma can be generated by prepulses, amplified spontaneous emission (ASE), or the rising edge of the driving laser pulse itself.

\footnotetext{
*malte.kaluza@uni-jena.de

Published by the American Physical Society under the terms of the Creative Commons Attribution 4.0 International license. Further distribution of this work must maintain attribution to the author(s) and the published article's title, journal citation, and DOI.
}

The relativistic hot electrons then propagate through the foil and form an electron sheath at its back, generating a strong electric space charge field. This field ionizes surface contaminations; the generated ions are accelerated in the target normal direction. In this regime, theoretical studies predict energies between several hundreds of megaelectronvolts and $1 \mathrm{GeV}$ [21]. However, these predictions could not be confirmed experimentally, the maximum proton energy was only increased from $58 \mathrm{MeV}$ [20] to $100 \mathrm{MeV}$ [19].

To identify ways for increasing the maximum proton energy $\mathcal{E}_{\mathrm{p}}$ with experimental parameters, e.g., laser pulse energy $E_{\mathrm{L}}$ or intensity $I_{\mathrm{L}}$, scaling laws have been derived with $\mathcal{E}_{\mathrm{p}} \propto$ $E_{\mathrm{L}}^{\xi}$ or $\mathcal{E}_{\mathrm{p}} \propto I_{\mathrm{L}}^{\xi}, \xi$ being the exponent of such power laws. For the TNSA regime, some models predict a rootlike scaling $\mathcal{E}_{\mathrm{p}} \propto I_{\mathrm{L}}^{0.5}[22,23]$, determined by the ponderomotive potential of the laser. Other models consider a constant conversion efficiency of laser energy into hot electrons [21] or derive the scaling from the electron temperature only [24], leading to different $\xi$. Experimentally, rootlike scalings $\mathcal{E}_{\mathrm{p}} \propto I_{\mathrm{L}}^{0.5}[25,26]$ and scalings of $\mathcal{E}_{\mathrm{p}} \propto I_{\mathrm{L}}^{0.8}$ [27] up to $\mathcal{E}_{\mathrm{p}} \propto I_{\mathrm{L}}^{1}$ [28] have been verified but only for limited parameter ranges. Generally, smaller $\xi$ were found for driving lasers with longer pulses ( $\tau_{\mathrm{L}} \geqslant 500 \mathrm{fs}$ ) and larger $\xi$ for $\tau_{\mathrm{L}} \leqslant 30 \mathrm{fs}$. The dependence of $\mathcal{E}_{\mathrm{p}}$ on $\tau_{\mathrm{L}}$ has also been investigated with picosecond- [26] and femtosecond-range pulses [21,29-31]. Here, a weaker dependence of $\mathcal{E}_{\mathrm{p}}$ on $\tau_{\mathrm{L}}$ than $E_{\mathrm{L}}$ was found, while the influence of $\tau_{\mathrm{L}}$ significantly changes with preplasma conditions [29]. However, all published scaling predictions show an unrestricted increase of $\mathcal{E}_{\mathrm{p}}$ with $E_{\mathrm{L}}$. On the other hand, the different scalings have only been verified experimentally for laser peak powers between 10 and 400 TW. A confirmation of the scalings to the petawatt or even multipetawatt level, where $\mathcal{E}_{\mathrm{p}}>$ $100 \mathrm{MeV}$ is predicted, has still not been reported [32-34], 


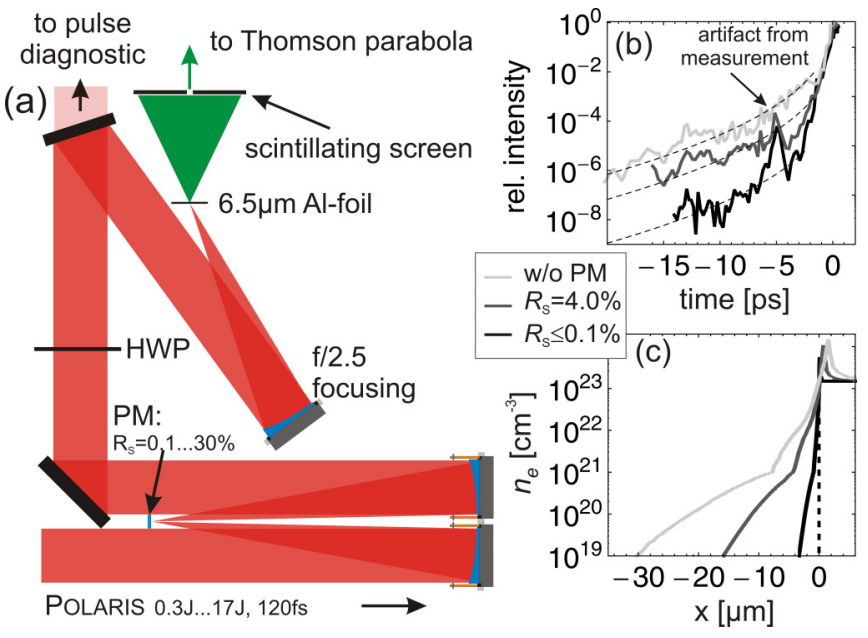

FIG. 1. (a) Layout of the experimental setup with the plasma mirror (PM), the $\lambda / 2$ waveplate (HWP), and the final $f / 2.5$ focusing. (b) Temporal intensity contrast measurements with and without PM with substrates of different $R_{\mathrm{S}}$. The feature at $-6 \mathrm{ps}$ corresponds to an artifact of the measurement. (c) Electron density distribution from hydrodynamic simulations for different contrast settings.

even though these systems have been operational for some time. Generally, the proton energies fall below the predicted values, suggesting that our understanding of the acceleration dynamics is still incomplete. The influence of additional experimental parameters needs to be included in our models.

In this paper, we present results from a study on laserproton acceleration from thin foils using $\tau_{\mathrm{L}}=120 \mathrm{fs}$ laser pulses. We investigated the dependency of $\mathcal{E}_{\mathrm{p}}$ on $E_{\mathrm{L}}$ to find and verify the aforementioned scalings. Furthermore, we varied the preplasma conditions at the target front. In the target normal direction $x$, the preplasma density can be approximated by $n_{\mathrm{e}}(x)=n_{0} \exp [-x / \mathcal{L}], \mathcal{L}$ being the preplasma scale length. By varying $\mathcal{L}$ experimentally, $\xi$ increased from 0.5 to 1 , underlining the importance of including $\mathcal{L}$ in the scalings. Furthermore, $\mathcal{E}_{\mathrm{p}}$ deviated from this power law behavior above a certain threshold $E_{\mathrm{L}}$, i.e., further increasing $E_{\mathrm{L}}$ did not further increase $\mathcal{E}_{\mathrm{p}}$. Our results may provide an explanation why proton energies $\mathcal{E}_{\mathrm{p}} \geqslant 100 \mathrm{MeV}$ have not been reported although predicted by the scalings.

\section{EXPERIMENTAL SETUP}

The experiments were performed at the POLARIS laser [35]. POLARIS delivered pulses (central wavelength $\lambda_{\mathrm{L}}=1030 \mathrm{~nm}$ ) with a full width at half maximum (FWHM) duration $\tau_{\mathrm{L}}=120 \mathrm{fs}$. The pulses were focused by an off-axis $F / 2.5$ parabolic mirror under an angle of $20^{\circ}$ onto the target [see Fig. 1(a)]. The focal spot had a $(7.9 \pm 0.5) \mu \mathrm{m}^{2}$ FWHM area, containing $20 \%$ of $E_{\mathrm{L}}$. Varying $E_{\mathrm{L}}$ between 0.3 and $17 \mathrm{~J}$ resulted in intensities of $0.5 \ldots 30 \times 10^{19} \mathrm{~W} / \mathrm{cm}^{2}$, corresponding to amplitudes of the normalized vector potential $a_{0}=\left[I_{\mathrm{L}} \lambda_{\mathrm{L}}^{2} /\left(1.37 \times 10^{18} \mathrm{Wcm}^{-2} \mu \mathrm{m}^{2}\right)\right]^{1 / 2}=2 \ldots 15$. A halfwave plate allowed the choice between $p$ or $s$ polarization. Here, $\tau_{\mathrm{L}}$ was measured online behind the compressor. Also, $E_{\mathrm{L}}$, near, and far field were recorded on every shot using the leakage through the last turning mirror [see Fig. 1(a)]. We used 6.5- $\mu \mathrm{m}$-thick aluminum foils, making TNSA the dominant mechanism here. For this target material, the preplasma formation has been extensively studied in hydrodynamic simulations [36] and experiments.

The temporal intensity contrast of POLARIS has recently been optimized significantly. At the current stage, femtosecond-short prepulses arriving nanoseconds [37] and picoseconds [38] before the main pulse are suppressed $<10^{-10} \times I_{\mathrm{L}}$. Furthermore, the ASE level is $<10^{-12} \times$ $I_{\mathrm{L}}[39,40]$. The rising edge of the pulses was steepened by a recollimating plasma mirror (PM). To realize different contrast conditions, PM substrates with residual reflectivities of $R_{\mathrm{S}} \leqslant 0.1 \%, R_{\mathrm{S}}=4 \%$, or $R_{\mathrm{S}}=30 \%$ were used. This led to a contrast enhancement between a factor of 2 and 600 [cf. Fig. 1(b)]. The preplasma conditions could be further varied by not using the PM but introducing an additional prepulse with $1.5 \times 10^{-5} \times I_{\mathrm{L}}$. This prepulse was generated before the second regenerative amplifier of POLARIS (see fig. 1 in Ref. [35]), therefore having the same duration and focal-spot size as the main pulse. The prepulse could arrive at $t=$ $-200 \mathrm{ps}$ or $t=-1 \mathrm{~ns}$ before the main pulse. To combine the different contrast settings in one parameter, one-dimensional (1D) hydrodynamic simulations using MULTI-FS [41] were carried out [Fig. 1(c)] based on the measured intensity contrast [Fig. 1(b)]. From these simulations, $\mathcal{L}$ was determined by fitting $n_{\mathrm{e}}(x)=n_{0} \exp [-x / \mathcal{L}]$ to the underdense region. Both the change of $\mathcal{L}$ with $E_{\mathrm{L}}$ and the spatial expansion of the preplasma were considered. At later times, the plasma expansion can no longer be assumed to be planar, which was accounted for by using a geometric expansion model (cf. Supplemental Material [42] ).

The proton energy spectra were measured with a Thomson parabola spectrometer using a microchannel plate as the detector. A scintillating screen was placed in front of the spectrometer. This screen was imaged onto a gateable chargecoupled device simultaneously measuring the proton beam profile, ensuring that the recorded spectrum corresponded to the central part of the proton beam [43].

\section{EXPERIMENTAL RESULTS}

$\mathcal{E}_{\mathrm{p}}$ as a function of $E_{\mathrm{L}}$ is shown in Figs. 2(a)-2(c) for different contrast settings. Figure 2(a) shows the scaling of $\mathcal{E}_{\mathrm{p}}$ for the long preplasma case, realized with the additional prepulse at $t=-1 \mathrm{~ns}$. The scale length was simulated to be $\mathcal{L}_{p}=29 \lambda_{\mathrm{L}} \pm 80 \%$ and $\mathcal{L}_{s}=24.5 \lambda_{\mathrm{L}} \pm 80 \%$ for $p$ and $s$ polarization, respectively. The scaling of $\mathcal{E}_{\mathrm{p}}$ with $E_{\mathrm{L}}$, as shown in Fig. 2(a), was fitted with $\xi_{p}=0.55 \pm 0.04$ and $\xi_{s}=0.62 \pm 0.02$. This almost rootlike scaling for both polarizations indicates that $\mathcal{E}_{\mathrm{p}}$ mainly follows the ponderomotive potential of the laser [22].

Figure 2(b) shows the $\mathcal{E}_{\mathrm{p}}$ scaling for the intrinsic POLARIS contrast, i.e., using neither PM nor prepulse. Here, the scale lengths were simulated to be $\mathcal{L}_{p}=3.5 \lambda_{\mathrm{L}} \pm 67 \%$ and $\mathcal{L}_{s}=$ $2.3 \lambda_{\mathrm{L}} \pm 67 \%$. For these contrast conditions, significantly larger exponents of $\xi_{p}=0.89 \pm 0.06$ and $\xi_{s}=0.95 \pm 0.08$ were found. However, $\mathcal{E}_{\mathrm{p}}$ followed these scalings only up to a certain threshold in laser energy of $7 \mathrm{~J}$, corresponding to an intensity of $I_{\mathrm{L}} \approx 1.1 \times 10^{20} \mathrm{~W} / \mathrm{cm}^{2}$. Above this threshold, the $\mathcal{E}_{\mathrm{p}}$ curve flattens, i.e., doubling $E_{\mathrm{L}}$ from 7 to $14 \mathrm{~J}$ only slightly 

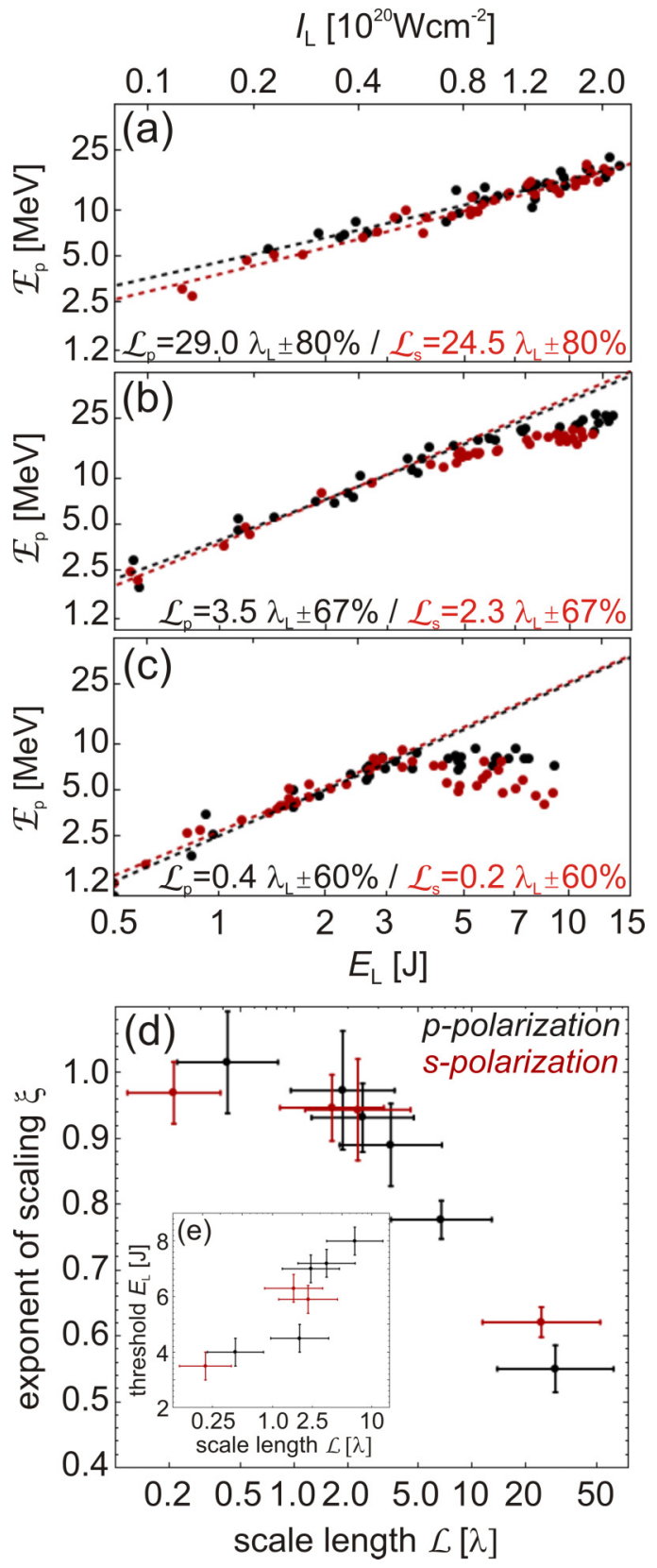

FIG. 2. (a)-(c) Double-logarithmic plots of the maximum proton energy $\left(\mathcal{E}_{\mathrm{p}}\right)$ as a function of the on-target laser energy $E_{\mathrm{L}}$ for $p$ (black) and $s$ (red) polarization and for different contrast settings: (a) without plasma mirror $(\mathrm{PM})$ but with a $1.5 \times 10^{-5} \times I_{\mathrm{L}}$ prepulse at $t=-1 \mathrm{~ns}$, (b) without PM, and (c) with PM using a substrate with $R_{\mathrm{S}} \leqslant 0.1 \%$. (d) Fitted exponent $\xi$ as a function of the preplasma scale length $\mathcal{L}$, which was simulated with MULTI-FS. (e) The threshold $E_{\mathrm{L}}$, above which a deviation of $\mathcal{E}_{\mathrm{p}}$ from the scaling law was observed, as a function of $\mathcal{L}$. See Supplemental Material [42] for additional data.

increased $\mathcal{E}_{\mathrm{p}}$ from 21 to $25 \mathrm{MeV}$, while $>40 \mathrm{MeV}$ would have been predicted.

Finally, Fig. 2(c) shows the $\mathcal{E}_{\mathrm{p}}$ scaling with a PM substrate with $R_{\mathrm{S}} \leqslant 0.1 \%\left(\mathcal{L}_{\mathrm{p}}=0.4 \lambda_{\mathrm{L}} \pm 60 \%\right.$ and $\mathcal{L}_{\mathrm{s}}=$ $\left.0.2 \lambda_{\mathrm{L}} \pm 60 \%\right)$. We found $\xi_{p}=1.02 \pm 0.08$ and $\xi_{s}=0.97 \pm$ 0.05 up to $E_{\mathrm{L}}=3.5 \mathrm{~J}\left(I_{\mathrm{L}}=5.5 \times 10^{19} \mathrm{~W} / \mathrm{cm}^{2}\right)$. Again, above this threshold, we could not further increase $\mathcal{E}_{\mathrm{p}}$ by increasing
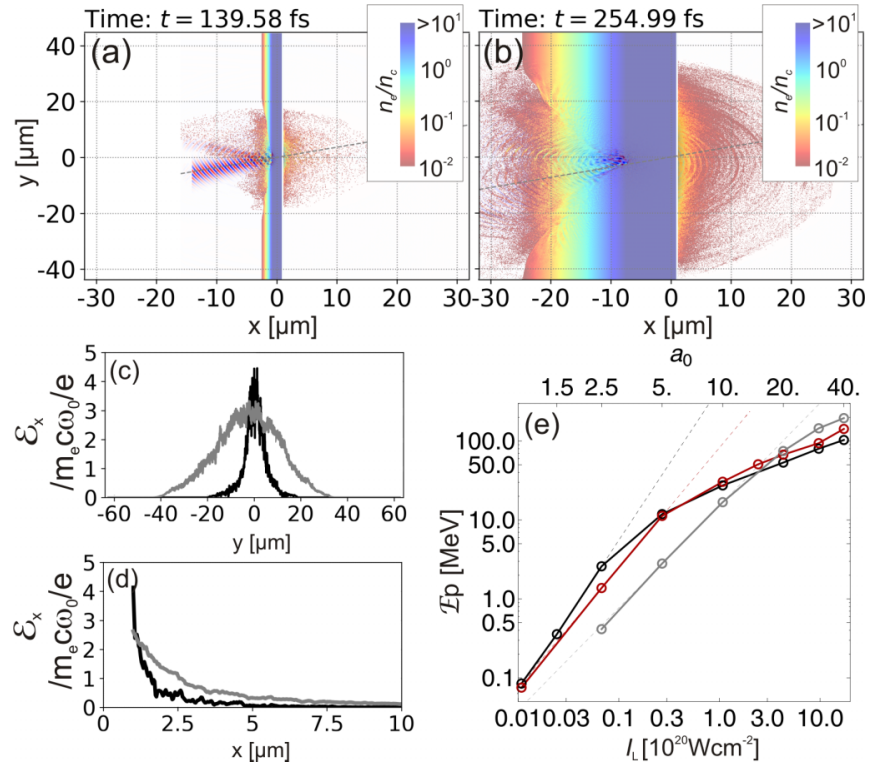

FIG. 3. Simulation results (I): Two-dimensional (2D) plots of the normalized electron density $n_{\mathrm{e}} / n_{\mathrm{c}}$ for $a_{0}=10$ and scale lengths of (a) $\mathcal{L}_{1}=0.25 \lambda_{\mathrm{L}}$ and (b) $\mathcal{L}_{2}=2.5 \lambda_{\mathrm{L}}$ at the time when the protons start to move. (c) Transverse lineout of the $x$ component of the electric field $\mathcal{E}_{x}$ on the target back (at $x=1 \mu \mathrm{m}$ ) for $\mathcal{L}_{1}$ (black) and $\mathcal{L}_{2}$ (gray). (d) Longitudinal lineout of $\mathcal{E}_{x}$ along the central axis (at $y=0$ ) for $\mathcal{L}_{1}$ (black) and $\mathcal{L}_{2}$ (gray). (e) Double-logarithmic plot of the simulated $\mathcal{E}_{\mathrm{p}}$ for $\mathcal{L}=0.25 \lambda_{\mathrm{L}}$ (black), $\mathcal{L}=0.5 \lambda_{\mathrm{L}}$ (red), and $\mathcal{L}=2.5 \lambda_{\mathrm{L}}$ (gray). The dashed lines are power law fits to $\mathcal{E}_{\mathrm{p}}$ at lower laser pulse energies.

$E_{\mathrm{L}}$. The change of $\xi$ as a function of $\mathcal{L}$ is summarized in Fig. 2(d) for $p$ (black) and $s$ (red) polarization. Here, $\xi \approx 0.5$ for longer scale lengths increases toward $\xi \approx 1.0$ when reducing $\mathcal{L}$. Furthermore, the $\mathcal{E}_{\mathrm{L}}$ threshold, above which $\mathcal{E}_{\mathrm{p}}$ dropped below the scaling, is shown in Fig. 2(e). For $120 \mathrm{fs}$ laser pulses, higher proton energies can only be reached with longer $\mathcal{L}$. For shorter $\mathcal{L}, \mathcal{E}_{\mathrm{p}}$ deviates from the scaling above a certain threshold. For the longest $\mathcal{L}$, no such threshold was found within our $\mathcal{E}_{\mathrm{L}}$ range. Furthermore, we did not observe any significant differences between $s$ and $p$ polarization. Finally, the behavior was similar for thinner targets $(2.4,1.5$, and $0.75 \mu \mathrm{m}$, cf. Supplemental Material [42]).

\section{NUMERICAL SIMULATIONS}

To explain our observations, we performed twodimensional particle-in-cell (2D-PIC) simulations with the PIC-ANTARES code (cf. Supplemental Material [42]). In the simulations, we varied $a_{0}$ between 1.5 and 40 and used different scale lengths $\mathcal{L}$ of $0.25,0.5$, and $2.5 \lambda_{\mathrm{L}}$. Exemplary simulation results are shown for $\mathcal{L}=0.25 \lambda_{\mathrm{L}}$ in Fig. 3(a) and for $\mathcal{L}=2.5 \lambda_{\mathrm{L}}$ in Fig. 3(b) at the time when the protons start to move away from the rear surface of the target.

For a comprehensive understanding of the $\mathcal{E}_{\mathrm{p}}$ scaling with $E_{\mathrm{L}}$, the evolution of the electric field $\mathcal{E}$ generated on the target rear surface and the position of the fastest protons within this evolving field must be considered. Only this allows a proper estimation of the maximum achievable proton energy. In Fig. 4, the central lineout of the longitudinal electric field $\mathcal{E}_{x}$ 


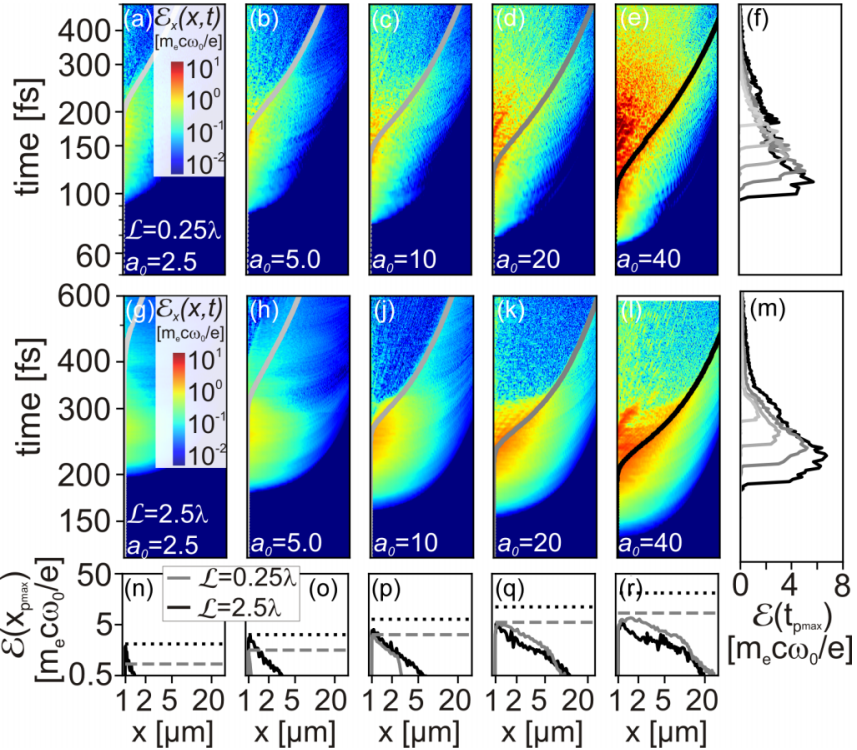

FIG. 4. Simulation results (II): Evolution of the longitudinal $\mathcal{E}_{x}$ field on the target rear side [cf. Fig. 3(d)] for different values of $a_{0}$ and scale lengths (a)-(f) $\mathcal{L}=0.25 \lambda_{\mathrm{L}}$ and $(\mathrm{g})-(\mathrm{m}) \mathcal{L}=2.5 \lambda_{\mathrm{L}}$. The trajectories of the fastest protons $x_{\mathrm{p} \text { max }}(t)$ are drawn as continuous lines. The lineouts correspond to the values of the longitudinal electric field $\mathcal{E}_{x}$ as experienced by the fastest proton with respect to (f) and (m) time and (n)-(r) space; for (n)-(r), a threshold defined by the maximum value of $\mathcal{E}_{x}(t)$ is shown as the dotted line for $\mathcal{L}=0.25 \lambda_{\mathrm{L}}$ and dashed line for $\mathcal{L}=2.5 \lambda_{\mathrm{L}}$. Please note that the maximum value can already be weakened due to the presence of accelerated protons and ions.

at $y \approx 0$ [cf. Fig. 3(d)] is plotted as a function of time for five different values of $a_{0}$ and for (a)-(f) $\mathcal{L}=0.25 \lambda_{\mathrm{L}}$ and (g)-(m) $\mathcal{L}=2.5 \lambda_{\mathrm{L}}$. In addition, the trajectory of the fastest proton $x_{\mathrm{p}_{\max }}(t)$ is shown by a solid line. Figure 4 shows not only that the maximum field strength increases with $a_{0}$ but also the lifetime of the field ( $\tau_{\mathrm{L}}$ was constant). With increasing $E_{\mathrm{L}}$, the laser is sufficiently intense for longer durations to produce hot electrons. Thus, the rear-surface electric field is generated earlier and maintains its large amplitude longer. In Fig. 4(a) $\left(a_{0}=2.5\right)$, we observe the onset of $\mathcal{E}_{x}$ at $\sim 100 \mathrm{fs}$, while in Fig. 4(e) $\left(a_{0}=40\right)$, the field already begins at $\sim 60 \mathrm{fs}[44]$. As a consequence, also the proton acceleration starts earlier, cf. the sequences in Figs. 4(a)-4(e). Starting at the lowest intensities [e.g., Figs. 4(a)-4(c)], increasing $E_{\mathrm{L}}$ first leads to both an increase of the maximum field strength experienced by the protons and of the duration of the acceleration process, cf. the temporal lineouts in Fig. 4(f). Consequently, $\xi$ is larger than expected when the maximum field strength alone is considered. However, if the intensity is increased further, the proton acceleration process starts earlier, and protons have already moved away from the target when $\mathcal{E}_{x}$ at the rear surface peaks. In Fig. 4(e), the fastest protons have already moved $\approx 5 \mu \mathrm{m}$, when $\mathcal{E}_{x}$ peaks at $t \approx 150$ fs. Since these protons no longer experience the highest $\mathcal{E}_{x}$, any further increase of $E_{\mathrm{L}}$ does not lead to a proportional increase of the acceleration. This is also seen in Figs. 4(n)-4(r), where the maximum field the protons experience is shown in comparison with a threshold defined by the maximum of $\mathcal{E}_{x}(t)$ (horizontal lines). For
$\mathcal{L}=0.25 \lambda_{\mathrm{L}}$ and $a_{0} \leqslant 5$, the fastest protons also experience the maximum field [Figs. 4(n)-4(o)]. From $a_{0}>5$ onwards, the field experienced by the fastest protons no longer reaches the maximum of $\mathcal{E}_{x}$; this difference increases with increasing $a_{0}$ [Figs. 4(p)-4(r)]. This ultimately leads to the maximum proton energy falling below the scaling. This supports our experimental observations [see Fig. 2(c)] and offers an explanation, why no protons with $\mathcal{E}_{\mathrm{p}}>100 \mathrm{MeV}$ have been detected with state-of-the-art petawatt lasers.

Furthermore, the dependence of $\xi$ on $\mathcal{L}$ can be explained by the longitudinal extension of $\mathcal{E}_{x}$. Figures 3(d) and 4 show that $\mathcal{E}_{x}$ spatially extends further for longer $\mathcal{L}$. This is due to the divergence angle of the hot electrons inside the target increasing linearly from $40^{\circ}$ to $70^{\circ}$ for $\mathcal{L}=1 \ldots 5 \mu \mathrm{m}[45,46]$. This larger divergence and the larger effective target thickness lead to a reduction in electron density and thus a reduced rearside field, also shown in Fig. 3. For $\mathcal{L}=0.25 \lambda_{\mathrm{L}}$ [Fig. 3(c), black], the transverse size of the field region is smaller and the peak is higher than for $\mathcal{L}=2.5 \lambda_{\mathrm{L}}$ (gray). The higher field in turn leads to a higher retaining force on the electrons and thus a reduced longitudinal extension. Hence, the peak-tolength ratio of the rear-side field could be varied by varying $\mathcal{L}$. This ultimately leads to the consequence that the fastest protons still experience the maximum of $\mathcal{E}_{x}$ even at higher $a_{0}$, cf. Figs. 4(n)-4(r) (gray lines).

In Fig. 3(e), the simulated $\mathcal{E}_{\mathrm{p}}$ scalings for the different preplasma scenarios are shown [44]. Here, $\xi$ increases for decreasing $\mathcal{L}$ in the low-intensity range. Toward higher intensities, we also observe the drop of $\mathcal{E}_{\mathrm{p}}$ below the initial scaling. For longer $\mathcal{L}$, this deviation occurs at higher intensities, agreeing very well with our experimental results. It should be noted that the interpretation of our experimental results does not depend on the absolute values of the scale length but only on the trend of the scale length when applying varying laser contrast conditions. The general trend of the correlation between scale length and the observed consequences for the accelerated proton pulses is clearly reproduced also with the modified simulations of the preplasma.

Finally, the time difference between the onset of the acceleration and the time when $\mathcal{E}_{x}$ peaks is crucial for the undisturbed scaling of $\mathcal{E}_{\mathrm{p}}$. A reduction of this difference would directly lead to a higher $\mathcal{E}_{\mathrm{p}}$ and could be achieved, for example, by shortening the pulse duration $\tau_{\mathrm{L}}$ or by manipulating the temporal shape $I_{\mathrm{L}}(t)$. Recently, Ziegler et al. [47] demonstrated experimentally that, by manipulating $I_{\mathrm{L}}(t), \mathcal{E}_{\mathrm{p}}$ could be significantly increased. Furthermore, an adjustment of $\mathcal{L}$ can mitigate the problem due to the increased longitudinal extension of the acceleration field. On the other hand, the time difference could also be reduced by either using a separate synchronized proton source like a double-stage approach [48] or by using cascaded acceleration regimes [49], in which protons from the front can be postaccelerated within the rear-side TNSA field. Finally, for targets with buried proton layers, delaying the injection of protons into the electric field could also be considered.

\section{CONCLUSIONS}

In summary, we have investigated the scaling of $\mathcal{E}_{\mathrm{p}}$ with $E_{\mathrm{L}}$ for $120 \mathrm{fs}$ high-power laser pulses. 2D-PIC simulations 
confirmed that, with increasing laser intensity, the back-side electric field is generated earlier and earlier. Hence, an increased laser energy leads to an increase in the electric field but also to an extension of the acceleration time and thus to a higher $\xi$ than expected. However, $\mathcal{E}_{\mathrm{p}}$ drops below this scaling above a certain threshold since, with increasing $E_{\mathrm{L}}$, the protons have already moved a significant distance away from the target when the acceleration field peaks. In this case, the protons no longer experience the maximum field strength, which ultimately leads to a limitation of the acceleration. This effect could be partly compensated by a proper choice of $\mathcal{L}$. A larger $\mathcal{L}$ increases the longitudinal extension of the field and thus counteracts this limitation. However, to overcome this limitation in general, the time difference between the onset of the acceleration and the peak of the field must be minimized. This can be done by optimizing the temporal laser pulse characteristics, by external injection of seed protons, or by delaying the generation of free protons by an adequate design of the back layers. Thus, our findings lead to a number of approaches to overcome the current limitations of the TNSA regime and could enable the acceleration of protons to energies $>100 \mathrm{MeV}$ in the near future, which would help bring envisaged applications closer to reality.

\section{ACKNOWLEDGMENTS}

The research leading to these results has received funding from LASERLAB-EUROPE (Grant Agreement No. 871124, European Union's Horizon 2020 research and innovation programme) and from the Bundesministerium für Bildung und Forschung (BMBF, Grants Agreement No. 03VNE2068D, No. 03Z1H531, No. 05K16SJC, No. 05K19SJC, No. 05P15SJFA1, and No. 05P19SJFA1).
[1] M. Roth, T. E. Cowan, M. H. Key, S. P. Hatchett, C. Brown, W. Fountain, J. Johnson, D. M. Pennington, R. A. Snavely, S. C. Wilks, K. Yasuike, H. Ruhl, F. Pegoraro, S. V. Bulanov, E. M. Campbell, M. D. Perry, and H. Powell, Fast Ignition by Intense Laser-Accelerated Proton Beams, Phys. Rev. Lett. 86, 436 (2001).

[2] J. C. Fernández, J. J. Honrubia, B. J. Albright, K. A. Flippo, D. C. Gautier, B. M. Hegelich, M. J. Schmitt, M. Temporal, and L. Yin, Progress and prospects of ion-driven fast ignition, Nucl. Fusion 49, 065004 (2009).

[3] M. Borghesi, D. H. Campbell, A. Schiavi, M. G. Haines, O. Willi, A. J. MacKinnon, P. Patel, L. A. Gizzi, M. Galimberti, R. J. Clarke, F. Pegoraro, H. Ruhl, and S. Bulanov, Electric field detection in laser-plasma interaction experiments via the proton imaging technique, Phys. Plasmas 9, 2214 (2002).

[4] L. Romagnani, J. Fuchs, M. Borghesi, P. Antici, P. Audebert, F. Ceccherini, T. Cowan, T. Grismayer, S. Kar, A. Macchi, P. Mora, G. Pretzler, A. Schiavi, T. Toncian, and O. Willi, Dynamics of Electric Fields Driving the Laser Acceleration of Multi-MeV Protons, Phys. Rev. Lett. 95, 195001 (2005).

[5] T. E. Cowan, J. Fuchs, H. Ruhl, A. Kemp, P. Audebert, M. Roth, R. Stephens, I. Barton, A. Blazevic, E. Brambrink, J. Cobble, J. Fernández, J.-C. Gauthier, M. Geissel, M. Hegelich, J. Kaae, S. Karsch, G. P. Le Sage, S. Letzring, M. Manclossi et al., Ultralow Emittance, Multi-MeV Proton Beams from a Laser Virtual-Cathode Plasma Accelerator, Phys. Rev. Lett. 92, 204801 (2004).

[6] S. Busold, A. Almomani, V. Bagnoud, W. Barth, S. Bedacht, A. Blazevic, O. Boine-Frankenheim, C. Brabetz, T. BurrisMog, T. E. Cowan, O. Deppert, M. Droba, H. Eickhoff, U. Eisenbarth, K. Harres, G. Hoffmeister, I. Hofmann, O. Jäckel, R. Jaeger, M. Joost et al., Shaping laser accelerated ions for future applications-the LIGHT collaboration, Nucl. Instrum. Methods Phys. Res., Sect. A 740, 94 (2014).

[7] P. Antici, M. Fazi, A. Lombardi, M. Migliorati, L. Palumbo, P. Audebert, and J. Fuchs, Numerical study of a linear accelerator using laser-generated proton beams as a source, J. Appl. Phys. 104, 124901 (2008).
[8] B. Dromey, M. Coughlan, L. Senje, M. Taylor, S. Kuschel, B. Villagomez-Bernabe, R. Stefanuik, G. Nersisyan, L. Stella, J. Kohanoff, M. Borghesi, F. Currell, D. Riley, D. Jung, C.-G. Wahlström, C. L. S. Lewis, and M. Zepf, Picosecond metrology of laser-driven proton bursts, Nat. Commun. 7, 10642 (2016).

[9] M. Barberio, M. Scisciò, S. Vallières, S. Veltri, A. Morabito, and P. Antici, Laser-generated proton beams for high-precision ultra-fast crystal synthesis, Sci. Rep. 7, 12522 (2017).

[10] M. Barberio, M. Scisciò, S. Vallières, F. Cardelli, S. N. Chen, G. Famulari, T. Gangolf, G. Revet, A. Schiavi, M. Senzacqua, and P. Antici, Laser-accelerated particle beams for stress testing of materials, Nat. Commun. 9, 372 (2018).

[11] S. V. Bulanov, T. Esirkepov, V. S. Khoroshkov, A. V. Kuznetsov, and F. Pegoraro, Oncological hadron therapy with laser ion accelerators, Phys. Lett. A 299, 240 (2002).

[12] K. Zeil, M. Baumann, E. Beyreuther, T. Burris-Mog, T. E. Cowan, W. Enghardt, L. Karsch, S. D. Kraft, L. Laschinsky, J. Metzkes, D. Naumburger, M. Oppelt, C. Richter, R. Sauerbrey, M. Schürer, U. Schramm, and J. Pawelke, Dose-controlled irradiation of cancer cells with laser-accelerated proton pulses, Appl. Phys. B 110, 437 (2013).

[13] D. Jahn, D. Schumacher, C. Brabetz, J. Dinga, S. Weih, F. Kroll, F. E. Brack, U. Schramm, A. Blazevic, and M. Roth, First application studies at the laser-driven LIGHT beamline: improving proton beam homogeneity and imaging of a solid target, Nucl. Instrum. Meth. A 909, 173 (2018).

[14] T. Esirkepov, M. Borghesi, S. V. Bulanov, G. Mourou, and T. Tajima, Highly Efficient Relativistic-Ion Generation in the Laser-Piston Regime, Phys. Rev. Lett. 92, 175003 (2004).

[15] H. Habara, K. L. Lancaster, S. Karsch, C. D. Murphy, P. A. Norreys, R. G. Evans, M. Borghesi, L. Romagnani, M. Zepf, T. Norimatsu, Y. Toyama, R. Kodama, J. A. King, R. Snavely, K. Akli, B. Zhang, R. Freeman, S. Hatchett, A. J. MacKinnon, P. Patel et al., Ion acceleration from the shock front induced by hole boring in ultraintense laser-plasma interactions, Phys. Rev. E 70, 046414 (2004).

[16] A. Pak, S. Kerr, N. Lemos, A. Link, P. Patel, F. Albert, L. Divol, B. B. Pollock, D. Haberberger, D. Froula, M. Gauthier, S. H. Glenzer, A. Longman, L. Manzoor, R. Fedosejevs, S. Tochitsky, 
C. Joshi, and F. Fiuza, Collisionless shock acceleration of narrow energy spread ion beams from mixed species plasmas using $1 \mu \mathrm{m}$ lasers, Phys. Rev. Accel. Beams 21, 103401 (2018).

[17] A. Henig, S. Steinke, M. Schnüerer, T. Sokollik, R. Hörlein, D. Kiefer, D. Jung, J. Schreiber, B. M. Hegelich, X. Q. Yan, J. Meyer-ter-Vehn, T. Tajima, P. V. Nickles, W. Sandner, and D. Habs, Radiation-Pressure Acceleration of Ion Beams Driven by Circularly Polarized Laser Pulses, Phys. Rev. Lett. 103, 245003 (2009).

[18] L. Yin, B. J. Albright, K. J. Bowers, D. Jung, J. C. Fernández, and B. M. Hegelich, Three-Dimensional Dynamics of Breakout Afterburner Ion Acceleration Using High-Contrast Short-Pulse Laser and Nanoscale Targets, Phys. Rev. Lett. 107, 045003 (2011).

[19] A. Higginson, R. J. Gray, M. King, R. J. Dance, S. D. R. Williamson, N. M. H. Butler, R. Wilson, R. Capdessus, C. Armstrong, J. S. Green, S. J. Hawkes, P. Martin, W. Q. Wei, S. R. Mirfayzi, X. H. Yuan, S. Kar, M. Borghesi, R. J. Clarke, D. Neely, and P. McKenna, Near-100 MeV protons via a laserdriven transparency-enhanced hybrid acceleration scheme, Nat. Commun. 9, 724 (2018).

[20] R. A. Snavely, M. H. Key, S. P. Hatchett, T. E. Cowan, M. Roth, T. W. Philips, M. A. Stoyer, E. A. Henry, T. C. Sangster, M. S. Singh, S. C. Wilks, A. MacKinnon, A. Offenberger, D. M. Pennington, K. Yasuike, A. B. Langdon, B. F. Lasinski, J. Johnson, M. D. Perry, and E. M. Campbell, Intense High-Energy Proton Beams from Petawatt-Laser Irradiation of Solids, Phys. Rev. Lett. 85, 2945 (2000).

[21] J. Schreiber, F. Bell, F. Grüner, U. Schramm, M. Geissler, M. Schnürer, S. Ter-Avetisyan, B. M. Hegelich, J. Cobble, E. Brambrink, J. Fuchs, P. Audebert, and D. Habs, Analytical Model for Ion Acceleration by High-Intensity Laser Pulses, Phys. Rev. Lett. 97, 045005 (2006).

[22] S. C. Wilks, W. L. Kruer, M. Tabak, and A. B. Langdon, Absorption of Ultra-Intense Laser Pulses, Phys. Rev. Lett. 69, 1383 (1992).

[23] P. Mora, Plasma Expansion into a Vacuum, Phys. Rev. Lett. 90, 185002 (2003).

[24] T. Kluge, T. Cowan, A. Debus, U. Schramm, K. Zeil, and M. Bussmann, Electron Temperature Scaling in Laser Interaction with Solids, Phys. Rev. Lett. 107, 205003 (2011).

[25] J. Fuchs, P. Antici, E. d'Humiěres, E. Lefebvre, M. Borghesi, E. Brambrink, C. A. Cecchetti, M. Kaluza, V. Malka, M. Manclossi, S. Meyroneinc, P. Mora, J. Schreiber, T. Toncian, H. Pépin, and P. Audebert, Laser-driven proton scaling laws and new paths towards energy increase, Nat. Phys. 2, 48 (2006).

[26] L. Robson, P. T. Simpson, R. J. Clarke, K. W. D. Ledingham, F. Lindau, O. Lundh, T. McCanny, P. Mora, D. Neely, C.-G. Wahlström, M. Zepf, and P. McKenna, Scaling of proton acceleration driven by petawatt-laser-plasma interactions, Nat. Phys. 3, 58 (2007).

[27] S. Ter-Avetisyan, P. K. Singh, K. F. Kakolee, H. Ahmed, T. W. Jeong, C. Scullion, P. Hadjisolomou, M. Borghesi, and V. Yu. Bychenkov, Ultrashort PW laser pulse interaction with target and ion acceleration, Nucl. Instrum. Methods Phys. Res. 909, 156 (2018).

[28] K. Zeil, S. D. Kraft, S. Bock, M. Bussmann, T. E. Cowan, T. Kluge, J. Metzkes, T. Richter, R. Sauerbrey, and U. Schramm, The scaling of proton energies in ultrashort pulse laser plasma acceleration, New J. Phys 12, 045015 (2010).
[29] M. Carrié, E. Lefebvre, A. Flacco, and V. Malka, Influence of subpicosecond laser pulse duration on proton acceleration, Phys. Plasmas 16, 053105 (2009).

[30] A. Flacco, F. Sylla, M. Veltcheva, M. Carrié, R. Nuter, E. Lefebvre, D. Batani, and V. Malka, Dependence on pulse duration and foil thickness in high-contrast-laser proton acceleration, Phys. Rev. E 81, 036405 (2010).

[31] S. Fourmaux, S. Buffechoux, B. Albertazzi, D. Capelli, A. Levy, S. Gnedyuk, L. Lecherbourg, P. Lassonde, S. Payeur, P. Antici, H. Pépin, R. S. Marjoribanks, J. Fuchs, and J. C. Kieffer, Investigation of laser-driven proton acceleration using ultra-short, ultra-intense laser pulses, Phys. Plasmas 20, 013110 (2013).

[32] F. Wagner, O. Deppert, C. Brabetz, P. Fiala, A. Kleinschmidt, P. Poth, V. A. Schanz, A. Tebartz, B. Zielbauer, M. Roth, T. Stöhlker, and V. Bagnoud, Maximum Proton Energy above 85 $\mathrm{MeV}$ from the Relativistic Interaction of Laser Pulses with Micrometer Thick $\mathrm{CH}_{2}$ Targets, Phys. Rev. Lett. 116, 205002 (2016).

[33] I. J. Kim, K. H. Pae, I. W. Choi, C.-L. Lee, H. T. Kim, H. Singhal, J. H. Sung, S. K. Lee, H. W. Lee, P. V. Nickles, T. M. Jeong, C. M. Kim, and C. H. Nam, Radiation pressure acceleration of protons to $93 \mathrm{MeV}$ with circularly polarized petawatt laser pulses, Phys. Plasmas 23, 070701 (2016).

[34] U. Schramm, M. Bussmann, A. Irman, M. Siebold, K. Zeil, D. Albach, C. Bernert, S. Bock, F. Brack, J. Branco, J. P. Couperus, T. E. Cowan, A. Debus, C. Eisenmann, M. Garten, R. Gebhardt, S. Grams, U. Helbig, A. Huebl, T. Kluge et al., First results with the novel petawatt laser acceleration facility in Dresden, J. Phys.: Conf. Ser. 874, 012028 (2017).

[35] M. Hornung, H. Liebetrau, S. Keppler, A. Kessler, M. Hellwing, F. Schorcht, G. A. Becker, M. Reuter, J. Polz, J. Körner, J. Hein, and M. C. Kaluza, $54 \mathrm{~J}$ pulses with $18 \mathrm{~nm}$ bandwidth from a diode-pumped chirped-pulse amplification laser system, Opt. Lett. 41, 5413 (2016).

[36] K. Eidmann, J. Meyer-ter-Vehn, J. T. Schlegel, and S. Hüller, Hydrodynamic simulation of subpicosecond laser interaction with solid-density matter, Phys. Rev. E 62, 1202 (2000)

[37] S. Keppler, R. Bödefeld, M. Hornung, A. Sävert, J. Hein, and M. C. Kaluza, Prepulse suppression in a multi-10-TW diodepumped Yb:glass laser, Appl. Phys. B 104, 11 (2011).

[38] S. Keppler, M. Hornung, R. Bödefeld, M. Kahle, J. Hein, and M. C. Kaluza, All-reflective, highly accurate polarization rotator for high-power short-pulse laser systems, Opt. Express 20, 20742 (2012).

[39] H. Liebetrau, M. Hornung, S. Keppler, M. Hellwing, A. Kessler, F. Schorcht, J. Hein, and M. C. Kaluza, High contrast, 86 fs, $35 \mathrm{~mJ}$ pulses from a diode-pumped, Yb:glass, double-chirpedpulse amplification laser system, Opt. Lett. 41, 3006 (2016).

[40] S. Keppler, A. Sävert, Jörg Körner, M. Hornung, H. Liebetrau, J. Hein, and M. C. Kaluza, The generation of amplified spontaneous emission in high-power CPA laser systems, Laser Photonics Rev. 10, 264 (2016).

[41] R. Ramis, K. Eidmann, J. Meyer-ter-Vehn, and S. Hüller, MULTI-FS - a computer code for laser-plasma interaction in the femtosecond regime, Comput. Phys. Commun. 183, 637 (2012).

[42] See Supplemental Material at http://link.aps.org/supplemental/ 10.1103/PhysRevResearch.4.013065 for additional experimental data, a discussion about the scale-length simulations, details on the 2D-PIC simulations, a discussion on the 
occurence of relativistic effects in the pre-plasma, and a discussion about the influence of hole-boring on the proton scaling [50-62].

[43] G. A. Becker, S. Tietze, S. Keppler, J. Reislöhner, J. H. Bin, L. Bock, F. E. Brack, J. Hein, M. Hellwing, P. Hilz, M. Hornung, A. Kessler, S. D. Kraft, S. Kuschel, H. Liebetrau, W. Ma, J. Polz, H. P. Schlenvoigt, F. Schorcht, M. B. Schwab et al., Ringlike spatial distribution of laser accelerated protons in the ultrahigh-contrast TNSA-regime, Plasma Phys. Control. Fusion 60, 055010 (2018).

[44] Note that explicit numbers are used here for an easier explanation only. Due to the lack of one spatial dimension in 2D-PIC simulations, the spatial extent of the fields, positions of the test protons, and the resulting proton energies can only be compared qualitatively with the experiment.

[45] A. Debayle, J. J. Honrubia, E. d'Humières, and V. T. Tikhonchuk, Divergence of laser-driven relativistic electron beams, Phys. Rev. E 82, 036405 (2010).

[46] V. M. Ovchinnikov, D. W. Schumacher, M. McMahon, E. A. Chowdhury, C. D. Chen, A. Morace, and R. R. Freeman, Effects of Preplasma Scale Length and Laser Intensity on the Divergence of Laser-Generated Hot Electrons, Phys. Rev. Lett. 110, 065007 (2013).

[47] T. Ziegler, C. Bernert, S. Bock, F.-E. Brack, T. E. Cowan, N. P. Dover, M. Garten, L. Gaus, R. Gebhardt, I. Goethel, U. Helbig, A. Irman, H. Kiriyama, T. Kluge, A. Kon, S. Kraft, F. Kroll, J. Metzkes-Ng, M. Nishiuchi, L. Obst-Huebl et al., Proton beam quality enhancement by spectral phase control of a PW-class laser system, Sci. Rep. 11, 7338 (2020).

[48] S. M. Pfotenhauer, O. Jäckel, J. Polz, S. Steinke, H.-P. Schlenvoigt, J. Heymann, A. P. L. Robinson, and M. C. Kaluza, A cascaded laser acceleration scheme for the generation of spectrally controlled proton beams, New J. Phys. 12, 103009 (2010).

[49] W. J. Ma, I. J. Kim, J. Q. Yu, I. W. Choi, P. K. Singh, H. W. Lee, J. H. Sung, S. K. Lee, C. Lin, Q. Liao, J. G. Zhu, H. Y. Lu, B. Liu, H. Y. Wang, R. F. Xu, X. T. He, J. E. Chen, M. Zepf, J. Schreiber, and X. Q. Yan, Laser Acceleration of Highly Energetic Carbon Ions Using a Double-Layer Target Composed of Slightly Underdense Plasma and Ultrathin Foil, Phys. Rev. Lett. 122, 014803 (2019).

[50] A. Andreev, T. Ceccotti, A. Levy, K. Platonov, and Ph. Martin, Divergence of fast ions generated by interaction of intense ultra-high contrast laser pulses with thin foils, New J. Phys. 12, 045007 (2010).

[51] G. A. Becker, M. B. Schwab, R. Lötzsch, S. Tietze, D. Klöpfel, M. Rehwald, H.-P. Schlenvoigt, A. Sävert, U. Schramm, M. Zepf, and M. C. Kaluza, Characterization of laser-driven proton acceleration from water microdroplets, Sci. Rep. 9, 17169 (2019).

[52] H. W. Powell, M. King., R. J. Gray, D. A. MacLellan, B. Gonzalez-Izquierdo, L. C. Stockhausen, G. Hicks, N. P. Dover, D. R. Rusby, D. C. Carroll, H. Padda, R. Torres, S. Kar, R. J. Clarke, I. O. Musgrave, Z. Najmudin, M. Borghesi, D. Neely, and P. McKenna, Proton acceleration enhanced by a plasma jet in expanding foils undergoing relativistic transparency, New J. Phys. 17, 103033 (2015).

[53] F. Dollar, C. Zulick, A. G. R. Thomas, V. Chvykov, J. Davis, G. Kalinchenko, T. Matsuoka, C. McGuffey, G. M. Petrov, L. Willingale, V. Yanovsky, A. Maksimchuk, and K. Krushelnick, Finite Spot Effects on Radiation Pressure Acceleration from Intense High-Contrast Laser Interactions with Thin Targets, Phys. Rev. Lett. 108, 175005 (2012).

[54] D. C. Carroll, O. Tresca, R. Prasad, L. Romagnani, P. S. Foster, P. Gallegos, S. Ter-Avetisyan, J. S. Green, M. J. V. Streeter, N. Dover, C. A. J. Palmer, C. M. Brenner, F. H. Cameron, K. E. Quinn, J. Schreiber, A. P. L. Robinson, T. Baeva, M. N. Quinn, X. H. Yuan, Z. Najmudin et al., Carbon ion acceleration from thin foil targets irradiated by ultra-high contrast, ultraintense laser pulses, New J. Phys. 12, 045020 (2010).

[55] B. Gonzalez-Izquierdo, R. J. Gray, M. King, R. J. Dance, R. Wilson, J. McCreadie, N. M. H. Butler, R. Capdessus, S. Hawkes, J. S. Green, M. Borghesi, D. Neely, and P. McKenna, Optically controlled dense current structures driven by relativistic plasma aperture-induced diffraction, Nat. Phys. 12, 505 (2016).

[56] Z. Lécz, J. Budai, A. Andreev, and S. Ter-Avetisyan, Thickness of natural contaminant layers on metal surfaces and its effects on laser-driven ion acceleration, Phys. Plasmas 27, 013105 (2020).

[57] H. Y. Wang, C. Lin, Z. M. Sheng, B. Liu, S. Zhao, Z. Y. Guo, Y. R. Lu, X. T. He, J. E. Chen, and X. Q. Yan, Laser Shaping of a Relativistic Intense, Short Gaussian Pulse by a Plasma Lens, Phys. Rev. Lett. 107, 265002 (2011).

[58] J. H. Bin, W. J. Ma, H. Y. Wang, M. J. V. Streeter, C. Kreuzer, D. Kiefer, M. Yeung, S. Cousens, P. S. Foster, B. Dromey, X. Q. Yan, R. Ramis, J. Meyer-ter-Vehn, M. Zepf, and J. Schreiber, Ion Acceleration Using Relativistic Pulse Shaping in Near-Critical-Density Plasmas, Phys. Rev. Lett. 115, 064801 (2015).

[59] Q. Liao, M. J. Wu, Z. Gong, Y. X. Geng, X. H. Xu, D. Y. Li, Y. R. Shou, J. G. Zhu, C. C. Li, M. Yang, T. S. Li, H. Y. Lu, W. J. Ma, Y. Y. Zhao, C. Lin, and X. Q. Yan, Enhanced laser proton acceleration by target ablation on a femtosecond laser system, Phys. Plasmas 25, 063109 (2018).

[60] Y. Yang, Z. Zhang, J. Jiao, C. Tian, L. Cao, Y. Wu, K. Dong, W. Zhou, Y. Gu, and Z. Zhao, Enhanced focusing of relativistic lasers by plasma lens with exponentially increasing density profiles, Phys. Plasmas 24, 063103 (2017).

[61] B. Aurand, S. Kuschel, O. Jäckel, C. Rödel, H. Y. Zhao, S. Herzer, A. E. Paz, J. Bierbach, J. Polz, B. Elkin, G. G. Paulus, A. Karmakar, P. Gibbon, T. Kuehl, and M. C. Kaluza, Radiation pressure-assisted acceleration of ions using multi-component foils in high-intensity laser-matter interactions, New J. Phys. 15, 033031 (2013).

[62] C. Scullion, D. Doria, L. Romagnani, A. Sgattoni, K. Naughton, D. R. Symes, P. McKenna, A. Macchi, M. Zepf, S. Kar, and M. Borghesi, Polarization Dependence of Bulk Ion Acceleration from Ultrathin Foils Irradiated by High-Intensity Ultrashort Laser Pulses, Phys. Rev. Lett. 119, 054801 (2017). 